\title{
ANKLE ANTAgonist CoActivation IN DOUBLE-SUPPORT PHASE OF WaLKING: StRoKe Vs Healthy SubJectS
}

\author{
Augusta Silva ${ }^{1}$, Andreia S. P. SOUSA ${ }^{1}$, CLÁudia Silva ${ }^{1}$, JoÃo ManUel \\ R. S. TAVARES ${ }^{2}$, RUBIM SANTOS ${ }^{3}$, FILIPA SOUSA ${ }^{4}$
}

${ }^{1}$ Escola Superior da Tecnologia de Saúde do Instituto Politécnico do Porto, Área Científica de Fisioterapia, Centro de Estudos de Movimento e Atividade Humana, Vila Nova de Gaia, Portugal

2 Instituto de Engenharia Mecânica e Gestão Industrial, Departamento de Engenharia Mecânica, Faculdade de Engenharia, Universidade do Porto, Portugal

${ }^{3}$ Escola Superior da Tecnologia de Saúde do Instituto Politécnico do Porto, Área Científica de Física, Centro de Estudos de Movimento e Atividade Humana, Vila Nova de Gaia,

Portugal

${ }^{4}$ Laboratório de Biomecânica do Porto, Faculdade de Desporto, Universidade do Porto,

Portugal

The authors have no conflicts of interest. 


\section{Abstract}

Introduction: Lesions at ipsilateral systems related to postural control at ipsilesional side, may justify the lower performance of stroke subjects during walking. Purpose: To analyse bilateral ankle antagonist coactivation during double-support in stroke subjects. Methods: Sixteen (8 females; 8 males) subjects with a first isquemic stroke, and twenty two controls (12 females; 10 males) participated in this study. The double support phase was assessed through ground reaction forces and electromyography of ankle muscles was assessed in both limbs. Results: Ipsilesional limb presented statistical significant differences from control when assuming specific roles during double support, being the tibialis anterior and soleus pair the one in which this atypical behavior was more pronounced. Conclusion: The ipsilesional limb presents a dysfunctional behavior when a higher postural control activity was demanded.

Keywords: Antagonist Coactivation ratio, Stroke, double support, walking, Leading limb, Trailing limb 


\section{Introduction}

Walking performance can be substantially affected after stroke (Milot et al. 2006; Achache et al. 2010), decreasing the ability to return to work, participate in the community or perform other daily activities (Higginson et al. 2006).

Most of the literature emphasizes the contralesional deficits in the contralesional side in relation to hemispheric lesion (CONTRA). It is well known that the CONTRA paretic muscles contribute differently in gait sub-phases when compared to healthy subjects. It has been recently hypothesized that stroke subjects may have postural control impairment also in ipsilesional side (IPSI), based on the possibility of cortico-reticular system lesion in stroke involving the territory of middle cerebral artery (Silva et al. 2012a; Silva et al. 2012b). However, few studies analysed the ipsilesional limb performance (Peterson et al. 2010; Rosa et al. 2014). This recent hypothesis justifies the possibility of bilateral involvement in stroke subjects.

During walking a consistent interlimb coordination has been demonstrated in subjects without neurological problems during step-to-step transition (double-support) (Sousa et al. 2012a; Sousa et al. 2012b). This functional connection between limbs (Hall et al. 2011; Anderson and Pandy 2003; Sousa et al. 2012a; Sousa and Tavares 2012) is supported by studies that found strong crossed effects of group II fibers in motorneurons pools (Corna et al. 1996) and by the role of reticulospinal system (with IPSI disposal) (Schepens and Drew 2004). Changes in the function of reticulospinal system during walking can be analysed through postural control behavior of soleus muscle (SOL), when this muscle acts to provide body support. Actually, it has been demonstrated that subjects with middle artery territory stroke present dysfunctional behavior of this muscle in the IPSI when its action is related to body support (Silva et al. 2012b). In fact in a study developed by Sousa et. al., 2013, a negative influence of IPSI SOL during loading response, as leading limb (LEAD), over forward propulsive, as trailing limb (TRAIL), muscles of CONTRA was found (Sousa et al. 2013). 
The dynamic relationship between limbs during walking, may also be analyzed through the levels of the antagonist coactivation ratio, related to functional position of each limb (TRAIL vs LEAD) and the subsequent role of each. In fact, during double support phase, TRAIL plantar flexors assume mainly the function of body support by SOL (McGowan 2008) and forward progression by GM action (Neptune et al. 2001; Anderson and Pandy 2003; Hall et al. 2011). LEAD limb is more related with smooth ground contact and weight acceptance, being the dorsi flexors the main agonists (Winter 1983). In this sense, it is important to evaluate the ankle antagonist coactivation when the LEAD limb is the IPSI and when the TRAIL is the CONTRA, but also when these roles are inverted. The present study aims to understand the behavior of each limb concerning the ankle antagonist coactivation between muscle pairs when assuming the role of TRAIL and LEAD.

\section{Methods}

\section{Participants}

Sixteen (8 females; 8 males) subjects with a first isquemic subcortical stroke, and twenty two healthy subjects (12 females; 10 males) participated in this study.

For the stroke subjects, the mean time since the injury was 26 months $(S D=9)$. All subjects suffered an injury in the region of the middle cerebral artery, more specifically in the internal capsule, which was confirmed by brain computerized axial tomography. All subjects included had a score lower than 34 on the Fugl-Meyer Assessment of Sensoriomotor Recovery After Stroke scale (Lamontagne et al. 2002a) and the capacity to perform gait without the use of orthoses.

The stroke subjects have also preserved the cognitive function to understand orders, which was confirmed by assessment using the Mini-Mental State Examination. All potential subjects with previous history of neurologic pathology (e.g., Parkinson, pontine and cerebellar lesions), sensory impairment, diabetes, thrombophlebitis, history of lower limb surgery, and any orthopedic or 
rheumatoid conditions interfering with walking capacity were excluded, as well as subjects under medication that could affect the motor performance.

Signals collected in the stroke group were compared with the obtained from sedentary healthy subjects, selected according to the same exclusion criteria applied to the stroke group. In addition, potential healthy subjects that had suffered any neurological disorder were excluded.

All participants gave their informed consent according to the Declaration of Helsinki.

\section{Instruments}

Ground reaction forces were collected from two force plates (BERTEC Corporation, USA, FP4060-10 and FP4060-08 models) connected to a signal amplifier (from BERTEC, AM6300 model). The activity of the ankle agonist muscles of TRAIL (namely gastrocnemius medialis (GM), SOL (Neptune et al. 2001)) and LEAD (namely tibialis anterior (TA) (Cappellini et al. 2006; Bonell et al. 2007)) were assessed through electromyography. The bilateral electromyographic signal of these muscles was monitored using a bioPLUX research wireless signal acquisition system (PLUX Wireless Biosignals S.A., Portugal). The signals were collected at a sampling frequency of $1000 \mathrm{~Hz}$ and were preamplified in each electrode and then fed into a differential amplifier with an adjustable gain setting (25-500 Hz; common-mode rejection ratio: 110 $\mathrm{dB}$ at $50 \mathrm{~Hz}$, input impedance of $100 \mathrm{M} \Omega$ and gain of 1000). Self-adhesive silver chloride electromyographic electrodes (Dahlhausen®, Köln, Germany) were used in a bipolar configuration with a distance of $20 \mathrm{~mm}$ between detection surface centers. The skin impedance was measured with an Electrode Impedance Checker (Noraxon USA Inc). The electromyography and force platform signals were analyzed with the Acqknowledge software (Biopac Systems Inc). 


\section{Procedures}

\section{Preparation}

Immediately before the electrode placement, the skin was prepared to reduce the impedance to a level equal or inferior to $5 \mathrm{~K} \Omega$.

Electrodes were placed in muscles midbelly according to anatomical references (Cheng et al. 2004; Klein et al. 2010). The ground electrodes were placed over each patella.

\section{Measurement}

After an explanation about the procedures, subjects were instructed to walk at their self-selected speed in a 8 meters walkway after a voice command. A self-selected walk speed was adopted since ankle plantar flexor muscles develop higher activity at this speed (Milot et al. 2008). To prevent fatigue, 1min rest between each trial was provided and the necessary repetitions were performed in order to obtain three valid trials. A trial was considered valid when at least one complete stance phase was collected by each force plate during the task. The electromyographic data were acquired from both lower limbs simultaneously.

\section{Data analysis}

The raw electromyographic signal and the ground reaction forces signal were processed using the Acqknowledge software. The raw electromyographic signal was filtered using a band-pass filter of 20 and $450 \mathrm{~Hz}$, processed using a root mean square (RMS) procedure (Lamontagne et al. 2002a; Billot et al. 2010), and normalized to mean signal over the entire gait cycle.

The signals from the force plates were also filtered, using a low-pass filter of $8 \mathrm{~Hz}$, and the force values were normalized to the weight of each subject (Turns et al. 2007). The double support phase was assessed through ground reaction forces. The beginning of double support during stance phase was defined as the interval where Fz of LEAD presents a value equal or higher than $7 \%$ of body weight, till the initiation of TRAIL swing phase (Sousa et al. 2012a; Sousa et al. 2013). The EMG activity of each muscle was assessed 
during double support in two conditions: (a) when the IPSI limb was the TRAIL and the CONTRA was the LEAD, and (b) when the CONTRA was the TRAIL and the IPSI limb was the LEAD. The ankle antagonist coactivation was calculated according to the following formula:

$$
\text { Antagonist coactivation }(\%)=\frac{\text { antagonist activity }}{\text { agonist }+ \text { antagonist activity }} \times 100
$$

\section{Statistics}

Using descriptive statistics, measures of central tendency (mean) and dispersion (standard deviation) for the magnitude of TA and SOL's electromyographic activity were calculated. Taking into account the small sample size of both groups $(n<10)$, it was assumed that the variables did not follow a normal distribution. Thus, the Wilcoxon Signed Rank Test was used to compare the magnitude of the muscles' activity and antagonist coactivation between both lower limbs. The confidence interval used was equal to $95 \%$, with a significance level of 0.05 .

\section{Results}

Ankle antagonist coactivation in IPSI limb changes according to its role on walking, LEAD or TRAIL. In fact, non-significant differences were observed in relation to healthy control when IPSI limb assumes the role of LEAD (Table 2). However, the antagonist coactivation between SOL and TA present statistical significant differences in relation to healthy control when in TRAIL position (Table 2).

Contrary to what was expected, CONTRA limb presented values more similar to the healthy controls when assuming the TRAIL position (Table 2). 


\section{Discussion}

The choice to study plantar flexor muscles (SOL and GM), instead of other proximal muscles, was based on the knowledge that, through sensorial feedback, they act as one functional unit (Cappellini et al. 2006) and are the main contributors to move the body forward (Grey et al. 2007; Lin et al. 2006; Neptune et al. 2008). However, while SOL contributes mostly to body support (McGowan 2008), GM acts mainly at providing forward progression (Neptune et al. 2001; Anderson and Pandy 2003; Hall et al. 2011). Based on the neurophysiologic mechanism of reciprocal enervation, TA was the antagonist selected to study coactivation process. It is also important to note that this study focus on the relation between pairs of muscles. Consequently, the results obtained could not reflect changes in individual muscle activity. In this sense, when both muscles present activation impairments, the antagonist coactivation in stroke subjects can be similar to the obtained in healthy subjects. However, this not mean that recruitment failure is not present, since this particular aspect was not evaluated.

\section{$\underline{\text { CONTRA limb antagonist coactivation }}$}

Globally the results of the present study reveal that non-significant differences were observed between CONTRA limb and CONTROL, contradicting previous studies reviewed in (Rosa et al. 2014). However, these results doesn't exclude possible muscle activation impairments in this limb (Olney and Richards 1996; Lamontagne et al. 2002b). Based on neurophysiology, it would be expected that when assuming LEAD position, the CONTRA limb would present ankle antagonist coactivation dysfunction as a result of a higher impairment in TA recruitment (agonist role) subsequent from the corticospinal lesion. However, the possible dysfunction of postural control system, also described in stroke subjects in this limb, can lead to a decreased activity of the antagonist activity (SOL and GM) (Sousa et al. 2013). This can explain the lack of differences observed in ankle coactivation comparing to CONTROL, when assuming the LEAD position. The same reasoning may explain findings obtained in CONTRA while assuming the LEAD position. In fact, both TA and GM are predominantly phasic muscles, and so both may 
express an atypical behavior in subcortical stroke in middle cerebral artery territory by the dependence of these muscles on the dorsolateral system. The SOL as a tonic muscle (Anderson and Pandy 2003; Hall et al. 2011; Neptune et al. 2001), depends of ventro- medial systems enervation. The possible lesion of both systems in this kind of stroke subjects GM, can justify the lack of differences in coactivation between these muscles comparing to CONTROL.

\section{$\underline{\text { IPSI limb antagonist coactivation }}$}

Despite the postural control demand associated to initial contact and loading response, non-significant differences were observed between IPSI limb and CONTROL. These results can be explained by the fact that TA have a major role in stability during this phase (Chow et al. 2012) whose activity is dependent mostly from unimpaired dorsolateral system control. When this limb was the TRAIL, this behavior was no longer similar to those evidenced by the CONTROL, being the TA/SOL the pair in which this atypical behavior was more pronounced. This result can be explained by the knowledge of ipsilateral disposal ventromedial system that as a strong influence over predominantly postural muscles like SOL (Figure 1). Taking this into consideration, the results of the present study seems to indicate that the IPSI limb postural control dysfunction, associated to a possible lesion of ventromedial systems, interferes with the body support function during forward propulsion. This hypothesis hasn't been questioned in previous studies about antagonist coactivation in stroke subjects as the changes observed in IPSI limb have been interpreted has a compensatory adaptative strategy (Lamontagne et al. 2000; Chow et al. 2012; Rosa et al. 2014). It is important to note that, since our study is dedicated to antagonist coactivation and not to individual muscle activity, future studies are required to confirm this possibility. In other hand, it should be highlighted that our criteria to selecting participants for our study based on vascular territory and lesion area hasn't been frequently considered in previous studies. Based on this, the differences obtained in the present study in relation to previous studies (Chow et al. 2012; Lamontagne et al. 2000) as to ankle coactivation can be related to this aspect. 
Our findings present a novel insight into post-stroke neuro-motor impairment, since also points out difficulties in IPSI limb possibly related to the vascular lesion.

\section{Conclusions}

The ipsilesional limb present a dysfunctional behavior when a higher postural control activity was demanded (as when assumed the trailing position). This dysfunction was more pronounced in soleus and tibialis anterior muscle pair as a result of a possible higher relation of soleus muscle with postural control demand.

\section{References}

Achache V, Mazevet D, Iglesias C, Lackmy A, Nielsen JB, Katz R, MarchandPauvert V. 2010. Enhanced spinal excitation from ankle flexors to knee extensors during walking in stroke patients. Clin Neurophysiol 121 (6):930-938. doi:10.1016/j.clinph.2009.12.037

Anderson FC, Pandy MG. 2003. Individual muscle contributions to support in normal walking. Gait Posture 17 (2):159-169

Billot M, Simoneau E, Van Hoecke J, Martin A. 2010. Coactivation at the ankle joint is not sufficient to estimate agonist and antagonist mechanical contribution. Muscle \& Nerve 41 (4):511-518. doi:10.1002/mus.21530

Bonell C, Tabernig C, Tabernig C Analysis of EMG temporal parameters from the tibialis anterior during hemiparetic gait. In: 16th Argentine Bioengineering Congress and the 5th Conference of Clinical Engineering, 2007. Journal of Physics: Conference Series, pp 1-7

Cappellini G, Ivanenko YP, Poppele RE, Lacquaniti F. 2006. Motor Patterns in Human Walking and Running. Journal of Neurophysiology 95 (6):34263437. doi:10.1152/jn.00081.2006

Cheng PT, Chen CL, Wang CM, Hong WH. 2004. Leg muscle activation patterns of sit-to-stand movement in stroke patients. Am J Phys Med Rehabil 83 (1):10-16. doi:10.1097/01.phm.0000104665.34557.56

Chow JW, Yablon SA, Stokic DS. 2012. Coactivation of ankle muscles during stance phase of gait in patients with lower limb hypertonia after acquired

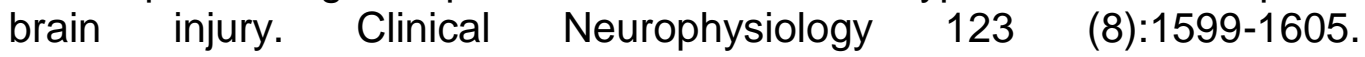
doi:http://dx.doi.org/10.1016/j.clinph.2012.01.006

Corna S, Galante M, Grasso M, Nardone A, Schieppati M. 1996. Unilateral displacement of lower limb evokes bilateral EMG responses in leg and foot muscles in standing humans. Exp Brain Res 109 (1):83-91

Grey MJ, Nielsen JB, Mazzaro N, Sinkjaer T. 2007. Positive force feedback in human walking. J Physiol $581 \quad(P t \quad 1): 99-105$. doi:10.1113/jphysiol.2007.130088 
Hall AL, Peterson CL, Kautz SA, Neptune RR. 2011. Relationships between muscle contributions to walking subtasks and functional walking status in persons with post-stroke hemiparesis. Clin Biomech (Bristol, Avon) 26 (5):509-515. doi:10.1016/j.clinbiomech.2010.12.010

Higginson JS, Zajac FE, Neptune RR, Kautz SA, Delp SL. 2006. Muscle contributions to support during gait in an individual with post-stroke $\begin{array}{lllll}\text { hemiparesis. } \mathrm{J} \text { Biomech } 39 & (10): 1769-1777 .\end{array}$ doi:10.1016/j.jbiomech.2005.05.032

Klein CS, Brooks D, Richardson D, Mcllroy WE, Bayley MT. 2010. Voluntary activation failure contributes more to plantar flexor weakness than antagonist coactivation and muscle atrophy in chronic stroke survivors. J Appl Physiol 109 (5):1337-1346. doi:10.1152/japplphysiol.00804.2009

Lamontagne A, Malouin F, Richards CL, Dumas F. 2002a. Mechanisms of disturbed motor control in ankle weakness during gait after stroke. Gait Posture 15 (3):244-255

Lamontagne A, Malouin F, Richards CL, Dumas F. 2002b. Mechanisms of disturbed motor control in ankle weakness during gait after stroke. Gait \& Posture 15 (3):244-255. doi:10.1016/s0966-6362(01)00190-4

Lamontagne A, Richards CL, Malouin F. 2000. Coactivation during gait as an adaptive behavior after stroke. J Electromyogr Kinesiol 10 (6):407-415

Lin PY, Yang YR, Cheng SJ, Wang RY. 2006. The relation between ankle impairments and gait velocity and symmetry in people with stroke. Arch Phys Med Rehabil 87 (4):562-568. doi:10.1016/j.apmr.2005.12.042

McGowan CP. 2008. Independent effects of weight and mass on plantar flexor activity during walking: implications for their contributions to body support and forward propulsion. J Appl Physiol 105:486-494

Milot M-H, Nadeau S, Gravel D, Bourbonnais D. 2008. Effect of increases in plantarflexor and hip flexor muscle strength on the levels of effort during gait in individuals with hemiparesis. Clinical Biomechanics 23 (4):415423. doi:10.1016/j.clinbiomech.2007.11.003

Milot MH, Nadeau S, Gravel D, Requiao LF. 2006. Bilateral level of effort of the plantar flexors, hip flexors, and extensors during gait in hemiparetic and healthy individuals. Stroke $37 \quad$ (8):2070-2075. doi:10.1161/01.STR.0000229900.88186.1a

Neptune R, Kautz A, Zajac E. 2001. Contributions of the individual ankle flexors to support, forward progression and swing initiation during normal walking. Journal of Biomechanics 34 (11):1387-1398

Neptune RR, Sasaki K, Kautz SA. 2008. The effect of walking speed on muscle function and mechanical energetics. Gait Posture 28 (1):135-143. doi:10.1016/j.gaitpost.2007.11.004

Olney SJ, Richards C. 1996. Hemiparetic gait following stroke. Part I: Characteristics. Gait \& Posture 4 (2):136-148. doi:10.1016/09666362(96)01063-6

Peterson CL, Hall AL, Kautz SA, Neptune RR. 2010. Pre-swing deficits in forward propulsion, swing initiation and power generation by individual muscles during hemiparetic walking. Journal of Biomechanics 43 (12):2348-2355. doi:10.1016/j.jbiomech.2010.04.027

Rosa MCN, Marques A, Demain S, Metcalf CD. 2014. Lower limb cocontraction during walking in subjects with stroke: A systematic review. 
Journal of Electromyography and Kinesiology 24 (1):1-10. doi:http://dx.doi.org/10.1016/j.jelekin.2013.10.016

Schepens B, Drew T. 2004. Independent and convergent signals from the pontomedullary reticular formation contribute to the control of posture and movement during reaching in the cat. J Neurophysiol 92 (4):22172238. doi:10.1152/jn.01189.2003

Silva A, Sousa A, Pinheiro A, Tavares J, Santos R, Sousa F. 2012a. Soleus activity in post-stroke subjects: movement sequence from standing to sitting. Somatossensory and Motor Research (in press)

Silva A, Sousa AS, Tavares J, Tinoco A, Santos R, Sousa F. 2012b. Ankle dynamic in stroke patients: agonist vs antagonist muscle relations. Somatossensory and Motor Research in press

Sousa AS, Santos R, Oliveira FP, Carvalho P, Tavares JM. 2012a. Analysis of ground reaction force and electromyographic activity of the gastrocnemius muscle during double support. Proc Inst Mech Eng H 226 (5):397-405

Sousa AS, Tavares JM. 2012. Effect of gait speed on muscle activity patterns and magnitude during stance. Motor Control 16 (4):480-492

Sousa ASP, Silva A, Santos R, Sousa F, Tavares JMRS. 2013. Interlimb Coordination During the Stance Phase of Gait in Subjects With Stroke. Archives of Physical Medicine and Rehabilitation 94 (12):2515-2522. doi:http://dx.doi.org/10.1016/j.apmr.2013.06.032

Sousa ASP, Silva A, Tavares JMRS. 2012b. Interlimb relation during the double support phase of gait: an electromyographic, mechanical and energy based analysis. Proceedings of the Institution of Mechanical Engineers, Part H: Journal of Engineering in Medicine 227 (3):327-333. doi:10.1177/0954411912473398

Turns L, Neptune R, Kautz S. 2007. Relationships between muscle activity and anteroposterior groud reaction forces in hemiparetic walking. Archives of Physical Medicine and Rehabilitation 88 (9):1227-1235

Winter D. 1983. Biomechanical motor patterns in normal walking. Journal of Motor Behavior 15 (4):302-330 